\title{
El día que cambió la historia del arma nacional de inteligencia ${ }^{1}$
}

LUISA FERNANDA LÓPEZ BOLAÑOS ${ }^{2, *}$

Resumen

El arma de Inteligencia Militar en Colombia se ha consolidado históricamente, desde su origen en 1964 hasta el día de hoy, como una de las unidades más importantes de las Fuerzas Militares por su compromiso con el lema "En Guardia por la Patria". En su 48 aniversario vale la pena resaltar, la honorable labor que ha desempeñado, con énfasis en el 2 de julio de 2008, que redefinió la historia de la inteligencia militar en Colombia, tras la ejecución de una exitosa operación, que cambió el rumbo tradicionalista, debido al sorprendente planeamiento y ejecución en la conocida Operación Jaque que, en su momento, protagonizó la prensa nacional e internacional.

Palabras clave: inteligencia militar, planeamiento, Operación Jaque, arma.

Clasificación JEL: N46, M12, H56, H57.

\section{Abstract}

The colombian intelligence corps has been historically consolidated, since its beginning until nowadays, as one of the most important corps in Military Forces due to its compromise with the slogan "On guard by the fatherland". It is worth noting in 48 intelligence anniversary, to stand out the honorable work which
${ }^{1}$ Artículo de investigación, correspondiente a la línea de Inteligencia y Contrainteligencia Estratégica.

${ }^{2}$ Profesional en Relaciones Internacionales y Estudios Políticos. Universidad Militar Nueva Granada.

•luisaf.lopezb@gmail.com.
Fecha de recepción: 10 de abril de 2018 .

Fecha de aceptación: 13 de mayo de 2018.

Para citar este artículo: López, L. (2018). El día que cambió la historia del arma nacional de inteligencia. Perspectivas en inteligencia, 10(19): 43-55. 
it has played, with emphasize in these July 2 that changed the corps tradition course because of amazing both planning and execution of the known Operation Jaque, which starred the national and international broadcast media by the moment.

Keywords: military intelligence, operation, planning, execution, Operation Jaque, corps.

JEL classification: N46, M12, H56, H57. 


\section{Introducción}

La Guerra Fría fue una era que no sólo determinó la "transición geopolítica" (Taylor, 2002, 81) en el escenario global -a mediados del siglo XX-, sino que también fundamentó las bases históricas de la Inteligencia Militar en Colombia, como consecuencia de la participación nacional en la Guerra de Corea, tras la división del Estado coreano en dos, originada en su participación como aliados en la Segunda Guerra Mundial, que dio origen a escaramuzas transfronterizas en el Paralelo 38, que dividía a la República de Corea (Corea del Sur) de la República Popular Democrática de Corea (Corea del Norte). Sin lugar a duda, las diferencias ideológicas entre el sur y el norte eran evidenciadas por el "tire y afloje" entre los ideales democráticos promulgados por occidente, principalmente por Estados Unidos, y el comunismo que tuvo como máximo representante a la Unión Soviética.

En 1950 Corea del Norte, apoyada por la República Popular China invadió a Corea del Sur, hecho que se convirtió en el primer conflicto armado de la Guerra Fría y encendió las alarmas internacionales sobre la amenaza que representaba el comunismo para la seguridad nacional e internacional, motivo por el cual la Organización de las Naciones Unidas (ONU), en cabeza de la potencia norteamericana, convocó a las naciones occidentales para que intervinieran en defensa de la democracia y la paz.

El entonces presidente de Colombia, Laureano Gómez, comprometido con el llamado de tropas y presidiendo a uno de los aliados de Estados Unidos, creó el Batallón de Infantería n. ${ }^{\circ}$, que tuvo como misión representar a los países hispanoamericanos en el Ejército de Naciones Unidas en Corea.

El armisticio de 1953, que terminó el conflicto y dio origen a una zona desmilitarizada para Corea, evidenció las técnicas de espionaje y sabotaje como características de las confrontaciones durante el período de la Guerra Fría, lo que particularmente dejó, como lección aprendida para Colombia, las falencias en el área de inteligencia, que dieron inicio a una historia que hoy en día es devanada sin precedentes.

Las clásicas técnicas de guerra han sido perfeccionadas al punto en que tanto las Fuerzas Militares como el enemigo, hacen uso de ellas ya sea en conflictos clásicos o asimétricos y, en este punto, vale la pena resaltar que estos últimos, no sólo marcan las tendencias contemporáneas de conflicto, sino que definen el contexto nacional. Es por ello que este artículo hará un reconocimiento a la honrosa labor de la comunidad de Inteligencia Militar, como una de las más importantes en las Fuerzas Armadas de Colombia en conmemoración de su XLVIII aniversario. 


\section{Perspectiva histórica de la inteligencia militar en Colombia}

Los cambios producidos por la Guerra Fría, en el contexto internacional manifestados en nuevos líderes y nuevas amenazas, definieron una novedosa situación de equilibrio de poder e "implicaron una formidable y peligrosa carrera de armamentos" (Taylor, 2002, 81) que exigía a los estados la creación y consolidación de estructuras capaces de garantizar la seguridad, mediante la recolección de información clave y ejecución de operaciones especiales contra el enemigo. Colombia no fue la excepción: años después de su participación militar en la Guerra de Corea, el Comando de la Fuerza seleccionó a un grupo de oficiales del Ejército Nacional, entre los cuales se destacó al Teniente Coronel Ricardo Charry Solano, para realizar un curso de inteligencia básica en Fort Halabird (EE.UU.) que tuvo como resultado "la creación del Destacamento de Inteligencia Militar del Comando de Ejército (1963)” (Historia de la Escuela de Inteligencia y C/I,1997, 16).

Al mismo tiempo, mientras el mundo enfrentaba la amenaza del comunismo, Colombia iniciaba una lucha contra su propia amenaza: la consolidación de las Fuerzas Armadas Revolucionarias de Colombia (FARC) quienes buscaban la toma del poder que, desde entonces, han librado una batalla para deslegitimar al Estado colombiano, convirtiéndose en el enemigo principal de la seguridad nacional e incluso regional.

En 1964, en medio de ese contexto de guerra, "el Comando del ejército creó el 02 de noviembre, el Batallón de Inteligencia y Contrainteligencia -BINCIante la necesidad de una unidad especializada" (Escuela de Inteligencia y Contrainteligencia, 2009, s.p.) que respondiera a los retos y desafíos que se imponían ante la seguridad. Esta fecha se constituyó emblemáticamente, en el día de conmemoración del Arma de Inteligencia del Ejército de Colombia, al oficializar el compromiso de la especialidad tanto con la preservación de los intereses nacionales como con la seguridad de sus habitantes.

La destacada labor del fallecido Brigadier General Ricardo Charry Solano hizo que, en 1970, se le atribuyera su nombre al BINCI y que, posteriormente en 1982, el Comando General de las Fuerzas Militares le diera el carácter de Unidad Especial al constituirse como Escuela, para consolidarse en 1985, por orden del "Ministerio de Defensa como Unidad Táctica el Batallón Escuela de Inteligencia y Contrainteligencia BG. Ricardo Charry Solano" (Historia de la Escuela de Inteligencia y C/I, 1997, 16).

Colombia fue el primer país en consolidar el Arma de Inteligencia en Latinoamérica, mediante la creación de un cuerpo de apoyo que oficializó su 
funcionamiento desde 1991, con la misión de producir inteligencia a través de la combinación de esfuerzos para obtener información y garantizar la seguridad y defensa nacional, tanto a nivel táctico como estratégico en medio de un contexto inquietante por el fortalecimiento de la concepción bélica a nivel internacional y el auge de la violencia al interior del país.

Desde entonces y comprometidos con la nación, la divisa azul oficializó en 1992 "el lema del arma de Inteligencia: CAVE PRO PATRIA ${ }^{3}$ (Historia de Escuela de Inteligencia y C/I, 1997, 24) que caracteriza no sólo la conmemoración histórica de la unidad especializada, sino que lidera el planeamiento y ejecución de misiones especiales con el objetivo de lograr el éxito operacional.

Cada 2 de noviembre y por 44 años, se conmemoró la constitución del Arma de Inteligencia como una de las más importantes de las Fuerzas Militares de Colombia, hasta el año 2008, en el que el 2 de julio se convirtió en la nueva fecha emblemática del arma. Durante esos 44 años, como reconocimiento a todos los que participaron en misiones de inteligencia se develó el Monumento de los Caídos -en 1992-, el Templo de la Inteligencia Militar, el Pasillo de Banderas y la Biblioteca Especializada en Inteligencia -en 1995-, afirmando que los miembros de la divisa azul permanecieron y aún continúan En guardia por la Patria contra las amenazas que "se encuentran cada vez más asociadas a fenómenos transnacionales como el terrorismo o las redes de criminalidad organizada" (Arcos, 2010,13) desde su conformación hasta nuestros días.

La Guerra Fría fue un período determinante para la consolidación de sistemas de inteligencia a nivel global, como estructuras capaces de impedir que el comunismo se expandiera al punto de convertirse en un elemento desestabilizador de la democracia-de acuerdo con los pronósticos occidentales-, lo que en términos de política internacional evidenció la existencia de un mundo bipolar, que luchaba por la defensa de los derechos humanos y los ideales democráticos, al tiempo que proclamó el comunismo como la amenaza global del momento.

El bipolarismo que lideraba el contexto internacional, cuestionó sobre la preparación de los Estados para enfrentar amenazas, materia en la que Colombia mostraba un gran avance al haber constituido ya su Unidad de Inteligencia, ante los desafíos de las modernas dimensiones de conflicto. Sin embargo, durante el período post-Guerra Fría, cuando muchos creían que la

${ }^{3}$ En guardia por la patria. 
amenaza había desaparecido, surgieron nuevos elementos que amenazaban la estabilidad de los estados como el narcotráfico y, más adelante, el terrorismo, hechos que reclamaban la acción inmediata de Inteligencia Militar.

En Colombia, el arma de Inteligencia Militar ha sabido hacer frente a las amenazas mediante la coordinación de operaciones que combaten al enemigo, mediante la innovación permanente en técnicas, tácticas y procedimientos que garantizan la seguridad y defensa nacional. Prueba de ello está en las exitosas operaciones planeadas y ejecutadas por los miembros de Inteligencia del Ejército Nacional para contrarrestar las acciones de grupos terroristas e incluso narcoterroristas como las FARC.

\section{Inteligencia militar vs. conflicto}

El auge del narcotráfico durante la década de los 90 y el consecuente reconocimiento del terrorismo como amenaza mundial por la comunidad internacional-después de los atentados del 9/11-, han posicionado a Colombia como una de las naciones más relevantes en el contexto global y especialmente en el latinoamericano, con respecto a la noción de seguridad, lo que sin lugar a duda ha exigido que el Ejército Nacional tenga como factores decisivos para alcanzar el éxito operacional la formación, educación, entrenamiento y reentrenamiento de su Arma de Inteligencia.

La inteligencia militar ha tenido un rol determinante desde su creación, debido a la realidad del conflicto colombiano, que durante décadas ha intentado desestabilizar el Estado. Gracias a la precisión en las fases de concepción, planeamiento, alistamiento y ejecución de operaciones de inteligencia se ha logrado reducir la capacidad de acción del enemigo y la neutralización de sus principales cabecillas. Particularmente en el contexto colombiano, cuando se habla de enemigo se hace referencia a la organización narcoterrorista FARC, no obstante existen otros actores al margen de la ley que constituyen una amenaza para la seguridad nacional, contra quienes también van dirigidas las acciones de ataque y contraataque, como son el Ejército de Liberación Nacional (ELN) y las hoy en día denominadas bandas criminales (Bacrim).

El tipo de guerra desatado por estos grupos al margen de la ley es un claro ejemplo de guerra irregular, que en términos de Friedrich Von Der Heydte es "un conflicto armado en el que los destacamentos no son grandes unidades, sino grupos de acción pequeños y más pequeños, donde el resultado se obtiene en una gran cantidad de pequeñas operaciones" (1987, 3), que exige 
una efectiva acción de inteligencia para lograr reducir sus operaciones en el territorio nacional y evitar la expansión transfronteriza.

La Inteligencia Militar en Colombia se ha caracterizado, durante la historia del conflicto armado interno, por desarrollar importantes operaciones contra el narcotráfico y contra las actividades subversivas de los actores paramilitares, que recientemente se han unido conformando una sola amenaza contra la seguridad nacional. Estas operaciones han sido planeadas y ejecutadas bajo los principios de Inteligencia de "necesidad, idoneidad y proporcionalidad" (Manual EJC. 2-10-1 Restringido, 2009, 5) y consolidados en una exitosa estrategia, producto de la excelente labor para la búsqueda y análisis de información acerca de la posible situación y posibilidades del adversario.

Las labores ejecutadas por la Inteligencia Militar del Ejército Nacional han demostrado la importancia del arma en operaciones de contrainsurgencia, exigiendo un alto nivel en el desarrollo de buenas tácticas, las cuales "no deben suceder en secuencia sino simultáneamente” (Paul, 2010) para garantizar el detrimento de actividades insurgentes.

La Inteligencia se configura así en ese componente que puede llegar a determinar el resultado de operaciones, a partir del balance previo entre las buenas y malas tácticas de la fuerza en cada uno de sus niveles, bien sea estratégico, avanzado o de combate. De igual manera, es imperioso incluir la interdependencia entre las técnicas de inteligencia para la exitosa ejecución de estrategias; es decir, debe haber una articulación entre la inteligencia técnica, la humana, y contrainteligencia, con el fin de aplicar estratagemas direccionados al objetivo.

La labor de Inteligencia Militar en Colombia ha logrado resultados sorprendentes debido a la excelente coordinación entre técnicas, tácticas y procedimientos, demostrada en operaciones destacadas contra el narcotráfico como la Operación Sorpresa, ejecutada en el municipio de Paujil, Caquetá (1996) mediante la cual se "evidenció la administración de 16 cocinas, 11 laboratorios para la elaboración de cocaína y una pista de aterrizaje por parte de los frentes 14 y 15 de las FARC" (Guzmán, 2004, 80); y la Operación Picudo en el Cauca (1999) donde fue capturado Flavio Espitia Bermúdez, "jefe de una red de heroína en Cali, que tenía además fuertes lazos con el "Mono Jojoy” (Guzmán, 2004, 83).

La Operación Gato Negro, una de las más importantes, apuntó a la destrucción del "imperio coquero" (Guzmán, 2004, 97) de las FARC en Vichada y Guainía, al mando de alias "el negro Acacio", quien mantenía alianza con alias "Fernandinho" (narcotraficante brasileño), para desarrollar un gran comercio ilícito de material 
bélico y estupefacientes con importantes mafias de Venezuela y Brasil, por la importancia estratégica que representa la zona. Esta gran operación estuvo antecedida por importantes operaciones militares como Soledad y Broche de Oro, y un arduo trabajo de inteligencia para la elaboración del esquema de maniobra que integraba a las brigadas participantes en la línea operacional.

Han sido numerosas las operaciones ejecutadas no sólo contra las FARC, sino también contra el ELN y grupos de autodefensa, operaciones que han obtenido resultados significativos para la seguridad, pero muchas veces carentes del reconocimiento público anhelado por sus ejecuciones en cubierta. No obstante, el éxito alcanzado ha sido producto del prudente y dispendioso trabajo de la fuerza conjunta que desarrolla tareas secretas, con el objetivo de garantizar la estabilidad de la nación y su población. Entre los recuerdos de Inteligencia están la Operación Bolívar (2001), Tumaco III (2002) y Dique I (2003), sólo por mencionar algunas.

El narcotráfico no ha sido el único objetivo de la Fuerza, pues se han desarrollado importantes operaciones para neutralizar a los cabecillas de las FARC -principal enemigo del Estado-, con el fin de contrarrestar su accionar delictivo y violento en el país, con la realización de extorsiones así como de una de las actividades que más repudio causa a nivel nacional e internacional: el secuestro, considerado una de las principales estratagemas del enemigo para presionar al gobierno y a la población civil.

Durante la última década la Inteligencia Militar ha dado fuertes golpes contra el enemigo, que confirman el compromiso de la unidad con la consolidación de la seguridad colombiana y regional, dejando en alto el nombre delarma y resaltando su honorífica labor en el país. Historia que se ve glorificada en operaciones trascendentales que demuestran el éxito de un Arma de Inteligencia íntegra, que desarrolla tareas de rescate, capturas, control de fronteras e incautación de material ilícito, con el objetivo de subyugar el conflicto y preparar a Colombia para el tan anhelado escenario de paz.

Resulta interesante reconocer que la historia se ha construido tras importantes tareas secretas, en las que participa un gran grupo de oficiales, suboficiales y agentes de inteligencia que arriesgan su vida por lograr el éxito operacional, del que ha sido protagonista nuestra arma de Inteligencia. Más aún que, durante los últimos años, la Inteligencia Militar ha logrado resultados significativos que dividen su historia en dos y enaltecen su labor a nivel mundial. En plena era de la política de Seguridad Democrática, se lograron ejecutar dos grandiosas operaciones que tuvieron como resultado el debilitamiento del Sistema Rival 
$\mathrm{FARC}^{4}$ y las cuales se convirtieron en el antecedente de la magna Operación Jaque, la que sin lugar a duda dio un giro a la historia de Inteligencia del Ejército Nacional de Colombia, lo que motivó cambiar el día de conmemoración del arma del 2 de noviembre al 2 de julio.

Previamente al desarrollo de dicha operación, hubo una que se convirtió en pieza fundamental para la Inteligencia Militar: la Operación Tifón, en abril de 2007, que relata la hazaña del subintendente Jhon Frank Pinchao, quien tras "18 días sobreviviendo en la selva, llegó desnutrido y exhausto al municipio de Pacoa (Vaupés)" (Torres, 2008, 23) donde pudo confirmar que su plan de fuga era un éxito. Una vez estando en Bogotá, su información fue vital para el planeamiento y ejecución de las tareas de inteligencia en aras de rescatar a los demás secuestrados, a través de las siguientes operaciones militares.

La primera de ellas fue la Operación Elipse en la que, mediante un asombroso trabajo de inteligencia en febrero de 2008, se pudo identificar la ubicación de los secuestrados, tras la observación de los tres norteamericanos en el río Apaporis (Guaviare) por un sargento y dos soldados que estuvieron mimetizados con la selva para esperar el movimiento del enemigo. Posteriormente, en marzo de 2008, se ejecutó la Operación Fénix en Santa Rosa de Sucumbíos (Ecuador) provincia que limita con el departamento de Putumayo, que tuvo como resultado la baja del segundo cabecilla de las FARC, Raúl Reyes, golpes que resultaron decisivos para la siguiente operación del año.

\section{La operación magna de inteligencia militar}

Una vez alcanzados los exitosos resultados de las anteriores operaciones, era momento de ejecutar la que marcaría el nuevo rumbo del Arma de Inteligencia, aquella que se convirtió en el titular de los periódicos nacionales e internacionales, la que conmocionó al mundo con la noticia del rescate de la ex candidata presidencial Ingrid Betancourt, tres norteamericanos y 11 miembros de la Fuerza Pública que se encontraban bajo el poder de las FARC; la operación que sólo ha podido compararse con la Operación Chavín de Huántar (Perú) pero aun así sigue siendo la número uno de los "casos de fuerzas especiales en acción", de acuerdo con una de las cadenas de noticias más importantes del mundo,

\footnotetext{
4 Término articulado en el marco de la mesa de Inteligencia del Comité de Revisión Estratégica e Innovación (CRE- I) del Ministerio de Defensa Nacional, 2011, para mirar objetivamente la organización FARC como un sistema opuesto con el fin de diagnosticar integradamente cada uno de sus componentes
} 
BBC News World (2011) y, en definitiva, la operación que cambió la historia del arma al instituir, desde el 2009 en adelante, al 2 de julio como el nuevo día de conmemoración de la Inteligencia Militar en Colombia: la Operación Jaque.

La operación ha sido denominada como una "epopeya militar y un homenaje a los Derechos Humanos sin precedentes" debido a la excelente combinación de los principios de guerra expuestos por Sun Tzu y Clausewitz, que podrían ser definidos en uno sólo y es que, si bien la "guerra es la continuación de las relaciones políticas con otros medios, la mejor victoria es vencer sin combatir". Así lo demostró la proeza de los hombres de Inteligencia encargados de ejecutar el rescate, simulando una comisión internacional.

Fueron varios los días que el grupo de comando de la Operación Elipse ${ }^{5}$ estuvo siguiendo paso a paso la rutina de los secuestrados en el río Apaporis, detectando los cambios horarios en el desarrollo de actividades y rastreando el posicionamiento, los cuales eran elementos claves para el planeamiento estratégico que seguiría el curso en el escenario. Sin embargo, esta primera fase de planeamiento estuvo en algunas ocasiones estropeada por cambios de posicionamiento inesperados del enemigo que implicaban nuevos frentes de acción. La nueva estrategia contempló como plan maestro: el engaño electrónico, basado en la interceptación de comunicaciones, con el objetivo de entender la estructura del enemigo.

El éxito en la interceptación y des encriptación de las comunicaciones del enemigo, representó grandes retos y desafíos para la divisa azul, caracterizados por un estricto y total control de factores que no daba lugar a falla alguna, de lo contrario caería la operación indiscutiblemente, razón que le dio el carácter de máxima confidencialidad de todas las operaciones que se habían desarrollado en el territorio nacional hasta la fecha. Esta operación implicaba el golpe más fuerte para las FARC, pues los rehenes del frente primero del Bloque Oriental (Ingrid Betancourt, los tres norteamericanos y 11 miembros de la fuerza pública) a cargo de alias "César" y "Gafas" constituían el punto de dominación del enemigo para el gobierno, por su relevancia política y estratégica.

La operación combinó una excelente articulación de inteligencia técnica y humana, que terminó con la insigne victoria de la operación táctica. Se crearon los roles específicos para cada uno de los miembros desarmados que participaron

5 Denominación que se le dio a la etapa de planeamiento de la Operación Jaque y la aplicación de inteligencia para ejecutarla exitosamente. 
en la supuesta comisión internacional y responsables del encuentro con los 15 rehenes y dos guerrilleros, para enfrentar lo que se constituyó como el más grande reto de la Inteligencia Militar, una operación en la que la adrenalina de tantos días de trabajo corría por las venas de cada uno de los hombres que ejecutaban la operación, y de los que al otro lado del escenario se habían constituido como sus idearios y comandantes.

Fue entonces ese memorable 2 de julio de 2008, que se ejecutó la más grande de las operaciones: el engaño era un hecho, el aislamiento de las comunicaciones permitió el éxito en la fase de planeamiento de la comisión internacional y enalteció el despegue del avión que había sido previamente maquillado para la misión, el cual volaba con un grupo de ocho personas a cargo del vuelo entre ingenieros y pilotos, además de los roles creados como el australiano, el árabe, dos hombres de prensa, un médico y una enfermera, quienes llevaron a la libertad a los 15 rehenes y lograron neutralizar a los dos cabecillas.

Los emotivos y previos momentos al rescate, el encuentro de la "comisión internacional" con los rehenes y el arduo trabajo para convencer a los mismos que subieran a la aeronave que les producía cierto temor y desconfianza por el incierto destino al que serían conducidos, son desde entonces recordados como uno de los logros más importantes de la inteligencia militar en Colombia; esa fecha inolvidable dividió la historia en dos, al transformar la conmemoración histórica del arma del 2 de noviembre, cuando se originó el arma de inteligencia como pionera en Latinoamérica, al 2 de julio como uno de los más fuertes golpes contra las FARC y la operación de inteligencia mejor ejecutada en la historia nacional e internacional.

\section{Conclusiones}

El triunfo de la Operación Jaque, no sólo implicó el reconocimiento nacional de los distintos mandos a cada uno de los participantes en su ejecución, ni el reconocimiento internacional a la labor de inteligencia en Colombia. Más allá de eso, ha llevado a realizar la conmemoración cada 2 de julio desde el 2009 de la honorífica arma de Inteligencia de las Fuerzas Militares de Colombia, ejemplo de compromiso con la patria y garante de la seguridad y defensa nacional.

En el 48 aniversario del arma, se hace un reconocimiento a la labor de Inteligencia Militar, que durante el pasado y el presente se ha constituido como ejemplo para los escenarios regional y mundial, y que sigue "En Guardia por la Patria” para sus futuras operaciones en pro de la soberanía nacional y continuar 
liderando operaciones con el objetivo de lograr sorprendentes resultados en el ambiente operacional.

Vale la pena resaltar que el compromiso sigue siendo Colombia y asílo evidencian la Operación Camaleón (2010) en Calamar (Guaviare), mediante la cual se logró el rescate de tres oficiales de la Policía Nacional y un suboficial del Ejército nacional que se encontraban bajo el poder de las FARC; la Operación Sodoma en la que fue abatido el jefe del Bloque Oriental en la región de la Macarena, alias "el Mono Jojoy"; la Operación Odiseo en 2011, que terminó con la muerte del líder máximo de las FARC, Alfonso Cano, y uno de los miembros de su anillo de seguridad, alias "el Zorro", además de la captura del jefe de seguridad alias "el Indio Efraín”; y las más recientes ejecutadas en el 2012 Faraón y Armagedón.

Son innumerables las operaciones que han definido la historia y la labor de la Inteligencia Militar en Colombia, así mismo, innumerables los oficiales, suboficiales y agentes que entregan su vida para asumir la dispendiosa tarea que su arma les ha impuesto. Por eso hoy nos enorgullecemos de decir que, en sus 48 años de existencia, ha ejecutado operaciones exitosas que marcan la historia nacional y a una de ellas se debe la nueva fecha conmemorativa del arma, la honorífica Operación Jaque que permite celebrar, cada 2 de julio, la grandeza de nuestra divisa azul. 


\section{Referencias}

1. Arcos, R., Antón, J. (2010). Reservas de Inteligencia: Hacia una comunidad ampliada de Inteligencia. Revista de Análisis y Prospectiva. Recuperado de: https://www.researchgate. net/publication/276044211_Reservas_de_Inteligencia_hacia_una_Comunidad_ ampliada_de_Inteligencia.

2. Bin Laden Killed: Ten cases of specialforces in action (2011). Recuperado de: http://www. bbc.co.uk/news/world-13287133.

3. Brownfield, W. (2009). Ejército Nacional. Revista Ejército. Edición No. 144. Bogotá D.C. Julio.

4. Clark, D. (2006). The vital role of intelligence in counterinsurgency operations. US Army war college.

5. Clausewitz, V. K. (1832). Recuperado de: http://www.laureatum.com.ar/documentos/ delaguerra.pdf

6. Escuela de Inteligencia y Contrainteligencia BG. Ricardo Charry Solano (1977). Biografía Sr. BG. Ricardo Charry Solano. Bogotá.

7. Escuela de Inteligencia y Contrainteligencia BG. Ricardo Charry Solano (1997). Historia de la Escuela de Inteligencia y Contrainteligencia BG. Ricardo Charry Solano. Bogotá

8. Fuerzas Militares de Colombia (2009). Manual de Sección Segunda Restringido. Segunda Edición. Sección Publicaciones Ejército.

9. Guzmán, I., Muñoz, J. (2004). El gran Cartel, la verdadera historia de la fuente de financiación de los grupos terroristas en Colombia. CIRCA - DKF Fundación Círculo de Amistad colombo - alemán.

10. Padilla de León, F. (2009). Jaque... jOperación Perfecta! Revista Ejército. Edición No. 144. Bogotá D.C.

11. Paul, C., Clarke, C. (2010). Victory has a thousand fathers, sources of success in counterinsurgency. Rand Corporation.

12. Stavidris, J. G. (2009). Operación Jaque: Un año después. Revista Ejército. Edición No. 144. Bogotá D.C.

13. Sun Tzu (1910). The Art of war. Oriental Printed Books and MSS.

14. Taylor, P., Flint, C. (2002). Geografía Política, Economía - mundo, Estado - nación y Localidad. Madrid. Editorial Trama.

15. Torres Cuellar, J. C. (2008). Operación Jaque, la verdadera historia. Bogotá D.C. Editorial Planeta.

16. Uribe Vélez, Á. (2008). Aniversario 189 del Ejército Nacional. Revista Ejército. Edición No. 140. Bogotá D.C.

17. Von Der Heydte, F. A. (1987). La Guerra Irregular Moderna. Bogotá. Gir de Colombia Ltda. Recuperado de: https://es.scribd.com/document/340428035/VON-DER-HEYDTELa-Guerra-Irregular-Moderna 